\title{
Translator : scriptor, compilator, commentator,
} auctor

Un logiciel d'entraînement au travail autonome en traduction

Jean Vaché

\section{OpenEdition \\ Journals}

Édition électronique

URL : http://journals.openedition.org/asp/4389

DOI : 10.4000/asp.4389

ISSN : 2108-6354

Éditeur

Groupe d'étude et de recherche en anglais de spécialité

Édition imprimée

Date de publication : 1 mars 1993

Pagination : 403-410

ISSN : 1246-8185

Référence électronique

Jean Vaché, «Translator : scriptor, compilator, commentator, auctor », ASp [En ligne], 1 | 1993, mis en ligne le 11 mai 2014, consulté le 02 mai 2019. URL : http://journals.openedition.org/asp/4389; DOI : 10.4000/asp.4389

Ce document a été généré automatiquement le 2 mai 2019.

Tous droits réservés 


\title{
Translator : scriptor, compilator, commentator, auctor
}

\author{
Un logiciel d'entraînement au travail autonome en traduction
}

Jean Vaché

1 Quasi-spécialité des études de langue en France, voire originalité de notre système, la traduction constitue une activité privilégiée qui accompagne le «cursus» du futur linguiste tout au long de sa formation. Traduire, c'est bien sûr, de tous les exercices proposés aux néophytes comme aux étudiants confirmés, l'un des plus difficiles; en traduisant un texte d'une langue vers une autre, le traducteur fait bien plus que d'opérer ce simple transfert que signale l'étymologie; en signant l'état final de sa réflexion critique sur le texte de départ, le traducteur se constitue en quelque sorte en " auteur » d'un nouvel état du texte; et pour ce faire, il doit combiner art et artisanat.

2 Dans les premiers cycles du supérieur, l'enseignement de la traduction ne peut que constituer une simple initiation à l'art de traduire ; en effet, pour d'évidentes raisons, le nombre et la fréquence des travaux de traduction proposés actuellement aux étudiants restent largement symboliques. Or, comme dans le cas de tout savoir-faire, les techniques artisanales ne se transmettent pas si aucun entraînement sérieux n'est proposé.

3 Les manuels de traduction, et autres recueils de versions préparées, que nous pourrions recommander à nos étudiants, ont pour principal défaut comme les éditions bilingues de juxtaposer les textes de départ et d'arrivée. On peut difficilement exiger des apprenants la grandeur d'âme qui consisterait à cacher la traduction proposée ; les nécessaires étapes de réflexion et de travail sont ainsi malheureusement court-circuitées. Lire directement une traduction-modèle ne constitue nullement un entraînement; c'est comme si un apprenti-pianiste se contentait d'écouter l'enregistrement par Dinu Lipatti de la valse de Chopin qu'il étudie au lieu de se mettre carrément au travail, au piano. J'ai l'ambition, avec mon logiciel de "Travail autonome de traduction", de mettre les étudiants à leur clavier.

4 En complément d'un cours de traduction que j'assure en seconde année de DEUG d'anglais, je crée un volant d'exercices complémentaires que les étudiants peuvent découvrir soit chez eux, soit au laboratoire « Informatique pour tous » de mon université. 
J'ai conçu l'exercice de telle façon qu'il se déroule dans le temps en une série d'étapes successives, démultipliant en quelque sorte la tâche de l'étudiant, tout en la téléguidant. Chaque exercice comprend une série de documents et d'informations : le texte complet à traduire ; quelques indications sur l'œuvre, sur l'auteur, sur le contexte; une division du texte en sous-unités de traduction; des notes de longueur variable attachées à tel ou tel élément du texte; et une proposition de traduction. Mais l'étudiant ne découvre ces informations que dans le déroulement temporisé de l'exercice et sa progression se fait au rythme de quatre phases successives qui sont, succinctement décrites, les suivantes:

- une première phase d'étude de la compréhension globale du texte, avec repérage des difficultés sémantiques, consultation de dictionnaires électroniques, constitution d'une fiche électronique de travail personnel (définitions, synonymes, hypothèses de traduction)

- une seconde phase d'étude raisonnée de chacune des sous-unités de traduction, avec consultation des notes hypertextuelles d'aide à la traduction gravées dans le texte par l'enseignant créateur de l'exercice.

- une troisième phase de création d'un premier «brouillon » de traduction pour chacune des sous-unités, un assemblage des fragments de traduction suivi d'une mise au point et d'un état final de la traduction complète de l'ensemble.

- une quatrième et dernière phase de vérification par l'étudiant de sa traduction par confrontation juxtalinéaire avec celle proposée par l'enseignant qui a préparé l'exercice.

5 En fin de séance d'entraînement, un fichier au nom de l'étudiant contenant la trace de son travail, le temps qu'il a passé à l'exercice et ses remarques personnelles est créé aux fins de vérifications complémentaires éventuelles. Par exemple, dans mon cas, je lis ensuite ces exercices, les commente, les annote et les rends, sous forme imprimée, aux auteurs ; ces exercices ne sont pas notés. En cours d'exercice, le parcours du néophyte est balisé de la façon suivante.

6 En Phase Un, après avoir donné son nom (ou son pseudonyme), il découvre à son rythme le texte complet du document à traduire, prend connaissance des éléments de contexte mis à sa disposition, lit les indications données sur l'auteur, la période, l'œuvre. Il indique ensuite les termes qui dans le texte font problème pour lui, se constituant ainsi une liste de difficultés sémantiques. Enfin, grâce à cette liste, il ouvre automatiquement deux dictionnaires, l'un de définitions, l'autre de synonymes; définitions et listes de synonymes pouvant être copiées électroniquement dans un bloc-notes qui restera à la disposition de l'étudiant tout au long de l'exercice. Sa progression dans cette phase est linéaire.

7 En Phase Deux, le traducteur prend connaissance de la façon dont l'enseignant a divisé le texte complet en sous-unités. Selon les cas, et à la discrétion du créateur de l'exercice, la longueur d'une sous-unité peut varier d'un segment d'énoncé à un énoncé complet, à un groupe d'énoncés, ou même à un paragraphe. Le nombre de sous-unités qu'il doit examiner s'affiche sous ses yeux, avec le numéro de celle où il se trouve. Il peut aussi faire apparaitre le texte complet du passage à traduire, ou le cacher.

Le bloc-s, ou aide-mémoire, de définitions qu'il a constituées en Phase Un est aussi constamment à sa disposition. Mais surtout, c'est dans cette phase que le traducteur en herbe découvre les divers conseils de traduction que son enseignant a préparés pour lui et qui, grâce aux possibilités de l'hypertexte, apparaissent à l'écran lorsqu'il les consulte. À l'intérieur de cette phase, il lui est loisible de circuler dans n'importe quel ordre parmi les sous-unités. 
9 En Phase Trois, le traducteur devient plus actif ; les sous-unités défilent à nouveau, mais il s'agit maintenant de les traduire en utilisant tous les renseignements collectés dans les phases précédentes. Bloc-notes et texte complet sont disponibles. Ici aussi le balisage du parcours permet au traducteur de naviguer librement entre les diverses unités; en revenant en arrière, il peut effacer son brouillon précédent, rendant possibles les « repentirs ». Lorsque toutes les unités sont traduites, les divers fragments s'assemblent et forment un second état de brouillon, encore susceptible de modifications, « cosmétiques » aussi bien que radicales. Lorsque l'étudiant considère son travail comme terminé, il passe à la dernière phase, mais non sans avoir reçu le message suivant : « Si vous décidez de poursuivre l'exercice, vous ne pourrez plus revenir en arrière !».

10 La Phase Quatre est pour l'apprenti-traducteur le moment de vérité. Privé de la possibilité de repartir en arrière, il voit apparaître deux colonnes; celle de gauche contient «sa » traduction définitive; celle de droite, une traduction proposée par son enseignant. Son travail consiste maintenant à repérer les points forts et les points faibles de sa prestation en fonction de ce qui se trouve en vis-à-vis; c'est l'indispensable vérification qui, ici et de façon délibérée, est confiée à l'apprenant lui-même, au moins à ce stade (vous vous rappelez que la trace de son travail n'est en fait pas perdue ; elle peut être éventuellement récupérée par l'enseignant). C'est dans cette phase finale que se font sans doute les acquisitions les plus importantes. Je pense en effet qu'il est formateur que les erreurs de traduction (contresens, faux-sens, etc.) soient repérées dans sa propre traduction par la personne qui vient de faire l'exercice.

11 L'exercice est alors fini, mais il est encore possible d'enregistrer un message personnel destiné à l'enseignant (questions sur la traduction, commentaires divers, réclamations, etc.) avant de quitter le programme. Mes étudiants de seconde année de DEUG passent ainsi en moyenne 75 minutes sur une traduction de ce genre.

12 Je viens ici de décrire un instrument de travail d'un nouveau type, essentiellement différent du livre, qui permet de guider en quelque sorte pas à pas l'activité d'un apprenant au travers des diverses phases du processus créatif de l'acte de traduire. $\mathrm{Si}$, comme je propose de le faire, nous pouvons effectivement considérer que l'aboutissement de ce processus est un nouvel état du texte qui représente l'étape ultime de la réflexion critique sur le texte antérieur, nous ne sommes pas très loin de la description que donne Roland Barthes, dans S/Z (1975 : quatrième de couverture), de la gestion critique du texte antérieur.

13 Cette gestion était au moyen âge distribuée entre quatre rôles différents mais complémentaires, «dont aucun ne correspond à ce que nous appelons aujourd'hui le critique »:

14 «Le scriptor copiait, sans ajouter": l'apprenti ici ne recopie pas lui-même le texte, le « traitement informatique » du texte s'en charge bien évidemment pour lui.

15 "Ce que le compilator ajoutait ne venait jamais de lui » : l'apprenti est invité à « compiler " lui-même une somme d'information à des sources diverses, thesaurus ou dictionnaires ; il " ajoute » au texte une valeur qui ne vient pas de lui.

16 «Si le commentator mettait du sien dans le texte tuteur, c'était à seule fin de le rendre intelligible » : le maître intervient ici avec ses gloses au niveau hypertextuel pour rendre précisément « intelligible » ce qui avait a priori des chances de paraître inintelligible.

17 L'auctor enfin, risquait bien ses propres idées, mais en leur donnant toujours l'appui de ce qui s'était pensé hors de lui » : le traducteur devenu auteur risque effectivement bien ses 
propres idées en proposant sa traduction, mais en leur donnant toujours l'appui, par un double respect (respect de la lettre et de l'esprit du texte) de ce que qui s'est pensé hors de lui.

18 Ce que Barthes nous rappelle utilement sans doute ici, c'est que le lecteur/traducteur d'un texte essaye de jouer tous ces rôles à la fois. Mais nos étudiants n'en sont souvent pas encore à ce niveau de virtuosité rhétorique. En décomposant comme je l'ai fait dans mon logiciel d'entraînement les diverses étapes de l'acte de traduire, je désire tout simplement aider les étudiants à percevoir leur activité de traduction comme une activité cohérente et non comme une suite désordonnée de gesticulations tous azimuts. J'essaye aussi avec cet instrument de favoriser l'autonomie de chaque individu dans son travail individuel, laissant chacun s'exprimer dans le temps qui lui convient. Je propose donc cet outil à tous mes collègues, enseignants de traduction, qui ont ces mêmes préoccupations. Et je m'empresse de les rassurer; ce logiciel est conçu de telle sorte qu'aucune connaissance en programmation n'est nécessaire pour l'utiliser à créer des exercices.

\section{Fiche technique}

Travail Autonome de Traduction (TAT) permet d'une part à des enseignants de langue de préparer des exercices de traduction pour tous niveaux et toutes combinaisons de langues, et d'autre part à des étudiants de s'entraîner de façon autonome, chez eux ou en laboratoire, à la traduction de textes. Il se compose de deux piles Hypercard qui correspondent aux deux activités décrites ci-dessus :

\section{Entrée de TAT}

C'est ici que l'enseignant prépare l'exercice qu'il destine à ses étudiants. «Entrée de TAT » est un simple outil facilitant l'entrée de données : texte à traduire dans la « langue de départ ", texte traduit dans la langue d' "arrivée »; titre de l'exercice, informations sur le contexte, sur l'auteur, etc. C'est également ici que l'enseignant décide à sa guise du nombre et de la longueur des sous-unités de traduction; la création des notes hypertextuelles est aussi automatisée. Enfin, cet outil fabrique automatiquement un document « texte seul » destiné à renseigner la pile Matrice.

\section{Matrice de TAT}

21 Il s'agit d'une matrice vide à l'intérieur de laquelle sont importés les renseignements créés sous « Entrée de TAT ». L'importation laisse la matrice intacte et lance la génération automatique d'une pile « exercice » au nom du texte que l'on importe.

L'exercice proprement dit d'entraînement à la traduction comporte quatre phases principales qui sont :

- étude de la compréhension globale du texte; recherches sémantiques assistées par accès à des dictionnaires et thesaurus électroniques (pour certaines langues seulement), par accès à des glossaires spéciaux (pour d'autres langues)

- analyse du texte par sous-unités ; travail autonome avec assistance de notes hypertextuelles

- traduction partielle par sous-unités suivie de traduction complète

- enfin, vérification de la traduction par la méthode de la confrontation juxtalinéaire.

En fin de séance d'entraînement, un fichier contenant le résultat du travail, le temps passé sur l'exercice et les remarques de l'étudiant est créé à son nom à toutes fins utiles. 


\section{BIBLIOGRAPHIE}

Barthes, Roland. 1970. S/Z. Paris : Seuil.

Pour se procurer Travail autonome de traduction, écrire à l'Association informatique et nouvelle formation, 6 impasse des Lauriers, 34830 Clapiers.

\section{AUTEUR}

JEAN VACHÉ

Université Paul Valéry, Montpellier. jean.vache@univ-montp3.fr 